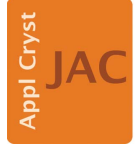

JOURNAL OF APPLIED CRYSTALLOGRAPHY

ISSN 1600-5767
Keywords: book review; solid electrolytes; allsolid-state batteries.

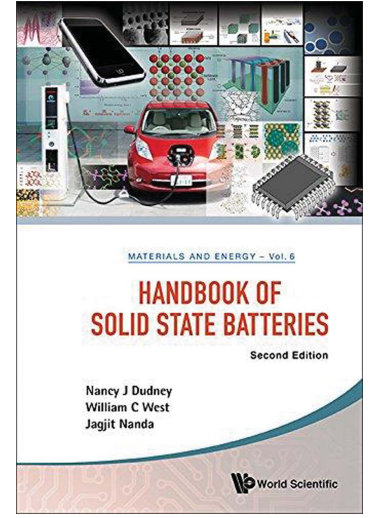

(C) 2016 International Union of Crystallography

\section{Handbook of Solid State Batteries, 2nd Edition. Edited by Nancy J. Dudney, William C. West and Jagjit Nanda. World Scientific, 2015. Pp. 836. Price (hardcover) GBP 155.00. ISBN 9789814651899.}

\author{
Virginie Viallet*
}

Université de Picardie Jules Verne, Laboratoire de Réactivité et Chimie des Solides, LRCS UMR 7314, 33 Rue Saint Leu, 80039 Amiens Cedex, and RS2E, Réseau Français sur le Stockage Electrochimique de l'Energie, CNRS FR\# 3459 , F 80039 Amiens, France. *Correspondence e-mail: virginie.viallet@u-picardie.fr

The title Handbook of Solid State Batteries speaks for itself, and this book represents an important contribution in the field of energy storage devices. Even though some review papers already exist on solid electrolytes and all-solid-state batteries, they are few and there has been so far no comparable book published in this field. That is why this new publication is of great interest for anyone wishing to explore this topic in greater detail. The aim of the contribution of Dudney et al. is to give an overview of the field of solidstate batteries, ranging from thin films to bulk all-solid-state batteries, and it provides a very good starting point to discover the field.

The book is quite complete, divided into three sections with a total of 22 chapters, most of them ending with outlook and perspectives, and is generally well written. In my opinion, an introductory chapter is missing, which could have provided some historical context and a discussion of the great advances and the barriers to overcome. Moreover, there are different expectations to fulfil between readers interested in microbatteries and those interested in massive or bulk solid batteries. The technologies are also quite different, and these two types of batteries deserve to be further addressed separately.

Part I, entitled Enabling Techniques and Fundamentals of Solid State Systems, contains six chapters (pp. 1-232). Part II, entitled Novel Solid Electrolyte Systems and Interfaces, contains ten chapters (pp. 233-589), and Part III, entitled Devices and 3D Architectures, contains six chapters (pp. 591-818). Part I might be misplaced and should be at the end as, at the beginning, no solid electrolyte has been detailed nor device described. At least, this part could have been separated into two parts. Chapters 1 and 6, dealing with solid electrolytes, should have been placed in Part II, leaving chapters 2, 3, 4 and 5, dealing effectively with analysis techniques, in Part I.

Chapter 1 defines what a solid electrolyte (also termed as superionic solid or fast ion conductor) is and addresses the fundamental aspects of ion transport in solid electrolytes. Microscopically, the ionic conductivity in crystalline solids is associated with structural changes above certain temperatures and/or is strongly dependent on the existence of defects. Lattice disorder and lattice defects are described in this chapter, as well as the structural features found to characterize the superionic solids. This chapter gives detailed descriptions of the mechanisms of ion transport and of the determination of diffusion coefficients by tracers, NMR or electrochemical methods, as well as the way to measure the ionic and the electronic conductivities and the methods of determining the transference numbers. Chapter 6 also deals with crystalline inorganic solid electrolytes and gives an interesting comparison between computer simulations and experiments. The purpose of this chapter is to describe examples of the use of first-principles calculations in the development of two families of materials: $\mathrm{Li}$ phosphates and thiophosphates and $\mathrm{Li}$ oxide garnets. The term 'first principle' in the so-called electronic structure methods implies a series of well developed approximations to the exact quantum-mechanical description of a material with $N_{\mathrm{e}}$ electrons and $N_{\mathrm{N}}$ nuclei. It is possible to study the structural parameters of stable and meta-stable structures and the approach can be extended to simulate ion mobilities. The reliability of the computer simulations is given by the agreement between the simulations and experiment.

Chapters 2, 3, 4 and 5 review different techniques relevant to the study of lithium and solid-state rechargeable batteries. 
Chapter 2 describes the potential applications, for lithium and thin-film batteries, of three in situ neutron techniques: neutron powder diffraction (NPD), neutron reflectivity (NR) and neutron depth profiling (NDP). NPD has been successfully utilized to analyse the chemical composition and crystal structure evolution occurring in electrodes or working batteries. NR is used to probe the interfacial structure evolution induced by Li insertion and extraction, and NPD is used to visualize directly the distribution and the flow of $\mathrm{Li}^{+}$ ions.

Chapter 3 provides a nice overview of various X-ray synchrotron-based spectroscopic techniques and their applicability to battery electrode materials. Detailed information about their local structure, oxidation state and crystalline order has been obtained, but one can regret that all the selected examples are related to conventional batteries and none to thin films or bulk all-solid-state batteries.

Chapter 5 comprehensively reviews recent examples where NMR has been successfully applied to both highly conducting solid electrolytes and selected anode materials to characterize $\mathrm{Li}$ ion diffusion. Different techniques are described, such as spin-lattice relaxation measurements with field gradient NMR techniques and/or field-cycling NMR measurements.

As suggested by its title, Part II is dedicated to novel solid electrolytes, in fact solid electrolytes in general, and interfaces. As a matter of fact, chapters 10 and 13, dealing with interfaces, could have been placed in Part III, dealing with devices and three-dimensional (3D) architectures. Moreover, they are not grouped but interspersed with those chapters dealing with solid electrolytes. The order in which the chapters on solid electrolytes are arranged appears not to be appropriate either. Part II begins with chapter 7 and the design of composite solid polymer electrolytes, while polymer electrolytes are described in the last two chapters, 15 and 16 . We comment on the different chapters following what could be an alternative way to arrange them.

Chapters 8, $911,12,14$ and 15 provide a comprehensive view of the state of the art of the different existing solid electrolytes, from a chemical or crystalline state point of view. Chapters 7 and 9 are more focused on the shaping of solid electrolytes, 7 for the design of solid polymer composites and 9 for thin films, and should have been placed at the end of Part II.

Chapter 12, rather short, gives a brief overview of the most promising superionic oxide (SCO) electrolytes, among the myriad of existing examples in the literature, including NASICON $\left[\mathrm{Li}_{1.3} \mathrm{Al}_{0.3} \mathrm{Ti}_{1.7}\left(\mathrm{PO}_{4}\right)_{3}\right.$ or LATP], perovskite $\left(\mathrm{Li}_{3 x} \mathrm{La}_{2 / 3-x} \mathrm{TiO}_{3}\right.$ or LLTO) and garnet $\left(\mathrm{Li}_{7} \mathrm{La}_{3} \mathrm{Zr}_{2} \mathrm{O}_{12}\right.$ or LLZO). Their electrochemical and chemical stabilities are discussed, in particular at lower potential against metallic Li. Interestingly the integration of SCO materials is discussed, with the numerous technical challenges including the fabrication of SCO membranes and the solid-state battery design. Chapter 12 is therefore a good introduction to Part II and also to Part III.

The counterpart of chapter 12 , chapter 11 provides a review of crystalline sulphide lithium ion conductors and their applications in solid-state batteries. Unlike oxide-based solid electrolytes, sulphides exhibit small grain boundary resistance (even after cold-pressing), which is an advantage for material processing and for fabricating large-scale bulk-type solid-state batteries. The structures of glass-ceramic electrolytes in the system $\mathrm{Li}_{2} \mathrm{~S}-\mathrm{P}_{2} \mathrm{~S}_{5}$ with or without $\mathrm{LiI}, \mathrm{LiBr}$ or $\mathrm{GeS}_{2}$ and in the system $\mathrm{Li}_{2} \mathrm{~S}-\mathrm{SiS}_{2}$, as well as crystalline thio-LISICON (lithium superionic conductors), are described and their sensitivity to air and moisture is also addressed. The high ionic conductivity and ease of densification make them attractive for solid-state batteries, but some challenges remain, the interfacial compatibilities of the sulphide-based solid electrolytes against the anode and the cathode being the most critical. As mentioned in chapter 12 (for oxides), the compatibility with metallic lithium is recognized as an issue and alternative anode materials have been developed, like the most frequently used lithium-indium ( $\mathrm{Li}-\mathrm{In})$ alloy (despite its cost), the $\mathrm{Li}-\mathrm{Al}$ alloy, $\mathrm{SnS} \cdot \mathrm{P}_{2} \mathrm{~S}_{5}$ amorphous electrodes and graphite anodes. Transition metal oxide- and phosphate-based cathodes are not the most suitable cathodes because of the interfacial reactivity of transition metals with sulphides, although this can be partially prevented by the coating of metal oxides such as $\mathrm{LiNbO}_{3}, \mathrm{Li}_{4} \mathrm{Ti}_{5} \mathrm{O}_{12}, \mathrm{LiTaO}_{3}$ and $\mathrm{Li}_{2} \mathrm{O}-\mathrm{SiO}_{2}$. Sulphidebased cathode materials are more promising because of their good interfacial compatibility, in particular for the Li-S technology, and more specifically for electrodes based on elemental sulphur and nanostructured $\mathrm{Li}_{2} \mathrm{~S}$ coated with $\mathrm{Li}_{3} \mathrm{PS}_{4}$.

Following on from chapter 12, chapter 14 is a succinct but rather complete overview of the glass and glass-ceramic sulphide and oxysulphide solid electrolytes, limited to the monovalent alkali cations $\mathrm{Li}^{+}$and $\mathrm{Na}^{+}$but also $\mathrm{Ag}^{+}$and $\mathrm{Cu}^{++}$. Tremendous progress has been made in these systems, in increasing the conductivities and improving their chemical and electrochemical stability. This, combined with a new highly scalable mechanochemical milling technique, has enabled the development of safe devices with high performance and a wide temperature range of operation.

Chapter 8 is entirely dedicated to fluoride ion conductors. Most commonly used in sensors, metal fluorides have attracted considerable interest in the development of energy materials, both as solid electrolytes and as active material in so-called fluoride ion batteries. Fast fluoride ion conductors based on fluorite and tysonite structures, as well as mixed compounds based on $\mathrm{Pb}, \mathrm{Sn}, \mathrm{Bi}$ and $\mathrm{Sb}$, are reviewed. Recent approaches to increase the conductivity, such as nanostructuring and tailoring of grain boundaries, are discussed. Unfortunately, no reference is made to the development of all-solid-state batteries incorporating these electrolytes. After ceramic oxides and crystalline or glass and glass-ceramic sulphide and oxysulphide solid electrolytes, polymers are also promising candidates for the development of all-solid-state batteries: chapters 15 and 16 are fully dedicated to their study.

Chapter 15 focuses on crystalline polymer electrolyte (PE), formed by alkali metal salts and poly(ethylene oxide). Although they were considered at first as insulators, the discovery of ionic conductivity in $\mathrm{PEO}_{6}: \mathrm{Li}_{X \mathrm{~F}_{6}}(X=\mathrm{P}, \mathrm{As}, \mathrm{Sb})$ 
and later in $\mathrm{PEO}_{8}: \mathrm{MAsF}_{6}(M=\mathrm{Na}, \mathrm{K}, \mathrm{Rb})$ expanded the field of these crystalline lithium PEs. A comprehensive study of the different factors influencing the conductivity is described, including the molecular weight of the polymer, the isovalent or aliovalent anion doping, the polymer doping, the size of the end groups, and the dispersity of polymer chain lengths. Electrochemical testing of two crystalline PEs in $\mathrm{Li}$ and $\mathrm{Na}$ rechargeable batteries is presented, demonstrating the successful operation of crystalline PEs in these two systems.

Chapter 16 reviews, in a very comprehensive and clear way, solvent-free systems as well as plasticized or gel electrolytes, beginning with the most studied one, poly(ethylene oxide), PEO, and its preparation methods. As polymers are complex and often heterogeneous materials, their investigation is challenging and requires a wide variety of methods, covering thermal analysis, rheology, vibrational techniques, diffraction techniques, scanning electron microscopy, conductivity measurements and NMR, which are briefly described. The implementation of polymer electrolytes in solid-state batteries is discussed, mainly for microelectric systems, and it appears that few companies are working towards polymer electrolyte batteries for vehicles. Also polymer oriented, chapter 7 presents the way to improve the mechanical properties of polymer electrolytes and the investigation of composite polymer electrolytes (CPEs). Progress has been made in the synthesis and characterization of CPEs filled with conductive $\left(\mathrm{Li}_{3} \mathrm{~N}\right.$, sulphide glasses in the system $\mathrm{Li}_{2} \mathrm{~S}-\mathrm{LiI}-\mathrm{B}_{2} \mathrm{~S}_{3}$, LLTO or LATP) or insulating $\left(\mathrm{TiO}_{2}, \mathrm{Al}_{2} \mathrm{O}_{3}, \gamma-\mathrm{LiAlO}_{2}, \mathrm{SiO}_{2}\right)$ fillers. The chapter also addresses the interfacial charge transport between the electrode and the solid electrolyte, the interfacial phenomena being distinguished by impedance spectroscopy. Modelling approaches have been developed to simulate and to design optimal composite architectures.

As mentioned earlier, chapters 10 and 13 are dedicated to interfaces, between solid electrodes and inorganic solid electrolytes: in general, in chapter 10 , and specifically between high-voltage cathodes with sulphide electrolytes in chapter 13. All-solid-state batteries contain many interfaces inducing different resistances. At the electrode/electrolyte interface, the resistance can increase because of the non-continuous contact of the rigid solids, possible volume expansion, or morphology changes of electrodes during $\mathrm{Li}^{+}$insertion or even caused by the $\mathrm{Li}$ ion charge itself. Chapter 10 describes the research and development for measuring and reducing the charge-transfer resistance at the electrode/inorganic solid electrolyte interface. Fundamentals as well as practical approaches are described, including interface modification and in situ formation. The interface modification by a surface coating, as mentioned in chapter 11, is effective for decreasing the charge-transfer resistance, due to the space-charge layer formed at the interface because of the difference of electrochemical potential of $\mathrm{Li}^{+}$in each phase and the mutual diffusion layer in the case of sulphide solid electrolyte. The concept of space-charge layer is also discussed in chapter 13. The effect is even more important when a high-voltage cathode, for instance $\mathrm{LiMn}_{2} \mathrm{O}_{4}, \mathrm{LiNi}_{1 / 3} \mathrm{Co}_{1 / 3} \mathrm{Mn}_{1 / 3} \mathrm{O}_{2}$ or $\mathrm{Li}_{1-x} \mathrm{CoO}_{2}$, comes into contact with a solid electrolyte, and is at the origin of the low power density of the corresponding batteries. A highly resistive lithium-depleted layer is formed on the electrolyte side of the interface, with an estimated thickness of around $10 \mathrm{~nm}$. One way to shield the sulphide electrolyte is to interpose an insulating buffer layer such as $\mathrm{Li}_{4} \mathrm{Ti}_{5} \mathrm{O}_{12}, \mathrm{LiNbO}_{3}, \mathrm{Li}_{2} \mathrm{O}-\mathrm{SiO}_{2}$ or $\mathrm{Li}_{2} \mathrm{SiO}_{3}$. Chapter 13 reviews requirements and coatings that allow optimized interfacial structures to be obtained. If they successfully reduce the interfacial resistance, there are still some challenges in the analysis of interfaces, as these thin space-charge layers are difficult to access by usual analytical methods. In the case of oxide solid electrolytes, as mentioned in chapter 10, a simple approach to reduce the charge-transfer resistance is to increase the number of electrochemically active sites at the interface of the two rigid solids and to promote adherence by in situ formation of the electrode material (Li metal or lithium insertion electrode material like $\mathrm{LiCoO}_{2}$ ).

Part III describes different devices. A chapter introducing the different existing all-solid-state batteries and the challenges of each system would have been useful. In a sense, chapter 18 could be considered as such, even if it is not evident from the title which mentions advancing conversion electrode reversibility with bulk solid-state batteries. This chapter gives a good understanding of solid-state batteries, by introducing thin-film batteries and the alternative bulk solid-state battery. It describes the architecture and then the different requirements and difficulties encountered, the main electrolytes developed, the solid-solid interfaces, and the importance of the preparation of the solid-state composite cathode, the anode being lithium metal or another composite. The chapter shows how the focus on bulk solid-state battery architecture has provided significant progress on reversible conversion chemistries, by considering the examples of $\mathrm{FeS}_{2}$ and $\mathrm{S}$, two of the more studied cathode conversion materials currently under investigation. To address the needs for practical, affordable and safe electric systems, in particular for electric vehicles, the technology of high-capacity conversion chemistries (e.g. $\mathrm{Li}-\mathrm{O}_{2}, \mathrm{Li}-\mathrm{S}, \mathrm{Li}-\mathrm{FeS}_{2}, \mathrm{Na}-\mathrm{S}$ ) is one of the three categories explored, along with multivalent intercalation and non-aqueous redox flow batteries. Further improvement in the energy density must be attained for solid-state batteries to be a viable alternative to conventional liquid cells, and methods for increasing it include the use of sulphur-carbon composites. New composite electrode processing technologies and the electrochemical utilization of the solid-state electrolyte are also described.

Chapter 17 is an inclusive and synthetic review of thin-film batteries, their fabrication methods, the different components of the cell, including cathode and anode materials, electrolytes, current collectors, and even protective encapsulations. The preparation and performance of thin-film batteries based on different combinations of cathode, electrolyte and anode materials are presented, as well as their different applications. As typical thin films or microbatteries share planar electrodes between components, they suffer from a fundamental tradeoff between power and energy density. The design of 3D battery electrodes, exhibiting nonplanar components, with 
higher surface area and the control over lithium ion diffusion distances, should solve this problem.

Chapter 20 discusses the strengths and weaknesses of 3D battery designs. If an impressive number of half-cells have been reported over the past decade, only a handful of complete solid-state 3D batteries have been realized, the limiting step being the solid electrolyte coating process. Several approaches are described, including extended thinfilm configurations, which produced the most successful semi3D or 3D batteries, interdigitated architectures, inverse opal geometries, aperiodic structures, and concentric tube designs. In the footsteps of this chapter, chapter 21 is dedicated to the electrochemical simulations of 3D battery architectures. In these systems, general electrochemistry is often described by a large set of partial differential equations (PDEs). Electrochemical modelling consists in solving these equations simultaneously for different parts of the battery cell. Mathematical models of $\mathrm{Li}$ ion batteries are described, in particular the concentrated solution theory developed by Newman and coworkers, which manages to provide a full electrochemical description of an Li ion battery, and the finite element method used to numerically solve second-order PDEs. However, so far, a very limited number of modelling studies have focused on 3D microbatteries. Computer simulations have been used to investigate the cell electrochemical behaviour, and also to guide optimization of material choice, electrode materials and electrolyte, and architecture or geometry.

Chapter 22 discusses the improvements in silver ion conducting solid-state electrolytes and silver solid-state batteries that have occurred over the past 20 years. As for other chemistries, three main types of ionic conductors are reported: crystalline ionic conductors, amorphous glass ionic conductors and doped polymers. Among the first discovered crystalline ionic conductors, silver iodide, AgI, is the most studied, and several strategies to increase the ion conductivity in the AgI-based materials are described. Ag conducting glasses can be obtained by dispersing silver salt, $\operatorname{Ag} X$ ( $X$ being a halide), in the glass former $A_{M} \mathrm{O}_{N}(A$ being a transition metal) and by using $\operatorname{Ag}_{2} Y$ ( $Y$ being a chalcogenide, $\mathrm{O}, \mathrm{S}$ or Se) as glass modifier. Silver doped polymer electrolytes are formed by combining a silver salt with a polar matrix. Most silver ion conducting solid electrolytes used in solid-state batteries include a matrix host material of $\mathrm{Ag}_{2} \mathrm{O}$ or $\mathrm{AgI}-\mathrm{Ag}_{2} \mathrm{O}$ materials. With very few exceptions, most silver ion conducting electrolytes have been used to produce primary batteries. The search for new cathodes and changes in the processing technique, like spark plasma sintering, as well as the study of polymer electrolytes may be possible to produce rechargeable silver solid-state batteries.

Chapter 19 addresses the approach of using multi-functional materials and designs to integrate energy storage, such as batteries, supercapacitors and capacitors, and structural or host platform components, such as covers, hatches, body panels, mounting structures or protective housings, to reduce overall system and volume. After having described the conventional or mono-functional approach, the authors discuss the various existing approaches, grouped into three main categories named conformable, embedded and structural devices. With fewer limitations but more challenging than the two others, the structural approach is more detailed and the development of structural batteries, capacitors, supercapacitors and pseudo-capacitors is illustrated with many examples.

The overall structure of the book could have been better optimized but, in most cases, the titles of the different chapters are suggestive and understandable. The book will thus be a great resource for students and researchers discovering the field. Despite its relatively high cost, I highly recommend the Handbook of Solid State Batteries to anyone interested in this emerging area of research. 